\title{
Advanced divertor concept design and analysis for HL-2M
}

\author{
G.Y. Zheng ${ }^{1}$, X.Q. Xu ${ }^{2}$, D.D. Ryutov ${ }^{2}$, T.Y. Xia ${ }^{3}$, X.R. Duan ${ }^{1}$, H.D. He ${ }^{1}$ and Y.D. Pan $^{1}$ \\ ${ }^{1}$ Southwestern Institute of Physics, Chengdu, People's Republic of China \\ ${ }^{2}$ Lawrence Livermore National Laboratory, Livermore, CA 94550, USA \\ ${ }^{3}$ Institute of Plasma Physics, Chinese Academy of Sciences, Hefei, People's Republic of China, \\ Email: zgy@swip.ac.cn
}

\begin{abstract}
HL-2M is a tokamak device that is under construction and will be put into operation in the near future. Based on the magnetic coil design of HL-2M, standard divertor, snowflake divertor and tripod divertor configurations have been designed. The potential properties of snowflake divertor configurations have been analyzed, such as the low poloidal field $\left(B_{p}\right)$ area around the X-point, the connection length, target plate and magnetic field shear. The linear peeling-ballooning (P-B) mode is studied by BOUT++ code for snowflake divertor configurations. According to the divertor configuration properties of HL-2M, asymmetric target plates have been concept designed to be compatible with the intended single null (SN) divertor configurations as well as double null (DN) divertor configurations. The SOLPS5.0 code is used to predict the details of the divertor plasma under the conditions of the divertor configurations noted above without impurities. This result shows that the peak heat load on outer target plate of the advanced divertor is about $40 \%$ of that of the standard divertor. But more power will be transported to the inner target plate of advanced divertor, and this will cause a higher peak heat load on the inner target plate. The advanced divertor will also have to work under low plasma recycling conditions with high particle temperature and low density in an open divertor target geometry. When the SN configuration changes to a DN tripod divertor configuration, most of the power exhaust is handled by the outer divertor target plates and the peak heat load on these is about $4.1 \mathrm{MW} / \mathrm{m}^{2}$ (with a power exhaust of 20MW). This range of optimized divertor configurations and target geometry will enable the study of advanced divertor physics and high performance plasmas in HL-2M tokamak.
\end{abstract}

Key words: HL-2M, advanced divertor, divertor target geometry, SOLPS5.0, heat load.

\section{Introduction}

$\mathrm{HL}-2 \mathrm{M}^{[1]}$ is a new medium-sized copper-conductor tokamak device under construction and due to be put into operation in the near future. Its aims include experiments to study high performance plasma physics and engineering relevant to ITER and some aspects of a fusion reactor. The total design heating power of HL-2M is $25 \mathrm{MW}$, and the heat load on the target plates can be roughly compared with other machines by $P_{S O L} / R(\mathrm{MW} / \mathrm{m})$ and the heat load width at the outer mid-plane $\left(\lambda_{q}\right)^{[2]}$, where $P_{S O L}$ is the power flowing into SOL zone and $R$ is the major radius. $P_{S O L} / R$ is $14 \mathrm{MW} / \mathrm{m}$ and $\lambda_{q}<1 \mathrm{~mm}$ (with $B_{p-\text { mid }} \sim 0.85 \mathrm{~T}$, where $B_{p-\text { mid }}$ is the poloidal field strength at the outside mid-plane) of HL-2M with $I_{P}=3 \mathrm{MA}$, both of which are close to those of ITER 
(16MW/m, $\left.\lambda_{q} \approx 1.0 \mathrm{~mm}\right)$. In order to enhance the flexibility and controllability of experiments, HL-2M is designed with demountable toroidal field (TF) coils, with poloidal field (PF) coils placed inside the TF coils. From an engineering point of view, when the PF coils are closer to the core plasma, their feedback control ability will be improved, permitting larger elongation, higher plasma pressure, etc.. Moreover, the current necessary in the PF coils to generate a second X-point of advanced divertor configuration (e.g. snowflake ${ }^{[3]}$ or tripod $^{[4]}$ divertor configurations) will be reduced. According to the plasma parameters, auxiliary heating power and equilibrium configurations proposed for HL-2M, the divertor physics including heat load mitigation using advanced divertor configurations will be one of the important missions associated with high performance plasma operation.

Standard divertor configurations are designed by using the equilibrium code CORSICA with a free-boundary Grad-Shafranov solver ${ }^{[5]}$. The PF coils and some example divertor configurations of HL-2M are shown in figure 1, in which the four types of divertor configurations have the same principle parameters (major radius $R=1.78 \mathrm{~m}$, minor radius $a=0.62 \mathrm{~m}$, plasma current $I_{P}=2.0 \mathrm{MA}$ and elongation $\left.k_{95}=1.5\right)$. The potential properties of the snowflake divertor configurations intended in HL-2M have been analyzed ${ }^{[4]}$. The results show that the snowflake divertor configurations have a larger low $B_{p}$ area around the primary X-point of the snowflake divertor, which may reduce the heat load on the target plates by fast cross-field convective heat transport around the low $B_{p}$ zone due to the higher local $\beta_{p}$. This results in a larger plasma-wetted area and longer connection length to broaden the heat load profile or to share the power between multiple divertor legs, especially when an ELM event occurs ${ }^{[6]}$. But the maximum plasma-wetted area is limited by an engineering constraint ${ }^{[7]}$ such that $\gamma_{\min } \approx F_{t} \sin \alpha$, where $\alpha$ is the angle of $B_{p}$ between the target plate and $F_{t}=B_{p} / B_{t}$ ( $B_{t}$ is the toroidal field). The value of $\gamma_{\min }$ near the target plate is here considered in divertor configurations and target geometry design and adjusted by changing the position of the two X-points. The stronger magnetic shear of snowflake divertors is also expected to be beneficial for the magneto-hydrodynamic instabilities in the edge region which could improve core/pedestal plasma confinement ${ }^{[8,9]}$. The linear P-B stability analysis is computed by the BOUT++ code for these divertor configurations. The results show that the snowflake-minus divertor configuration should reduce the linear growth rate of the P-B modes by lessening the bad curvature on the low field side with the second X-point.
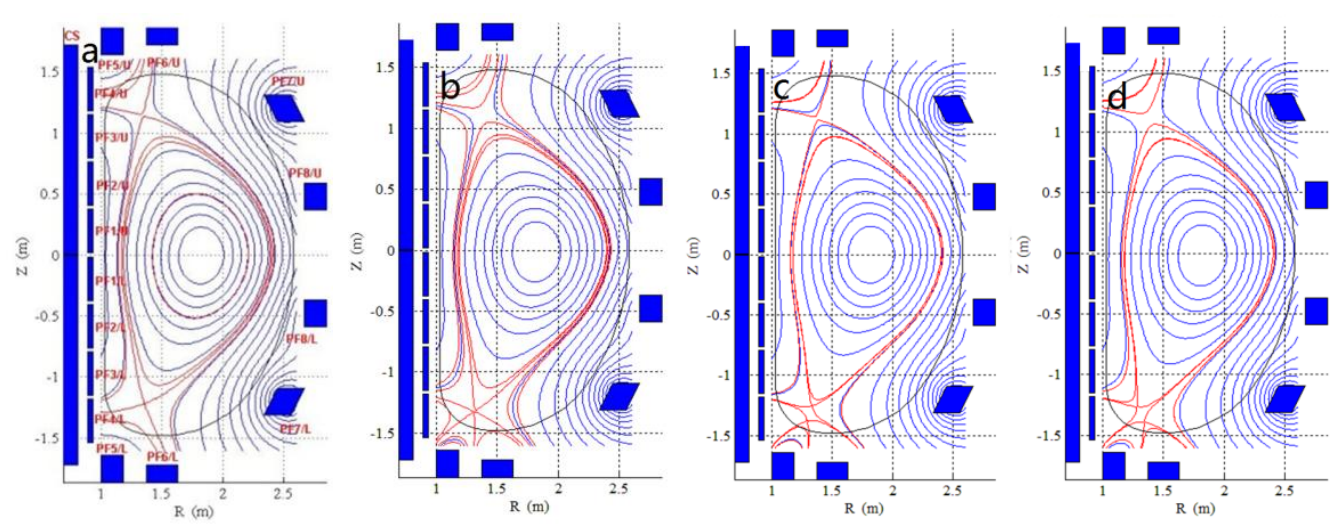

Figure 1. Example divertor configurations in HL-2M. The blue boxes show the positions of the PF coils. a)standard divertor configuration, b) exact snowflake divertor configuration, c) snowflake-plus divertor configuration, d) snowflake-minus divertor configuration. 
The snowflake divertor configurations shown above (for $I_{P}=2.0 \mathrm{MA}$ ) have two X-points with $d_{X}=15 \mathrm{~cm}\left(d_{X}\right.$ being the distance between the two X-points) to form a large low $B_{p}$ area which contains the two X-points and has $F_{p} \leq 0.1$ (where $F_{p}=B_{p} / B_{p-\text { mid }}$ ). Advanced divertor configurations with different plasma currents have also been investigated and are shown in figure 2. By taking advantage of the $B_{p}$ from the central solenoid (CS) coils, the position of the primary $\mathrm{X}$-point can be moved upward when the plasma current is reduced, and the second X-point can be held in its original position by adjusting the PF coil currents. So the distance between the two $\mathrm{X}$-points becomes longer, and the $F_{p} \leq 0.1$ zone around the two X-points separates into two small areas when $d_{X}$ is longer than $20 \mathrm{~cm}$. The effect of the second $\mathrm{X}$-point on the field structure at the primary X-point decreases and the configuration eventually loses the features of a snowflake divertor, becoming just that of a configuration with two separate X-points. The emerging configuration is different from a prototypical $\mathrm{X}$-divertor ${ }^{[10]}$ or the conceptually similar cusp divertor ${ }^{[11]}$ that relies on the use of specially arranged coils near the strike point. In this paper we will refer to this configuration with a long divertor leg and three outgoing branches of the separatrix a tripod configuration ${ }^{[3]}$. When the second X-point is designed to be close to the separatrix (i.e. with the distance from the separatrix normalized to outer mid-plane less than $\lambda_{q}$ ), then the power flows into the three branches. The plasma-wetted area will further increase due to modification of the angle between the branches and the target plate, while the appearance of the second X-point above the target plate may also usefully decrease the particle flow to the core region from the target.
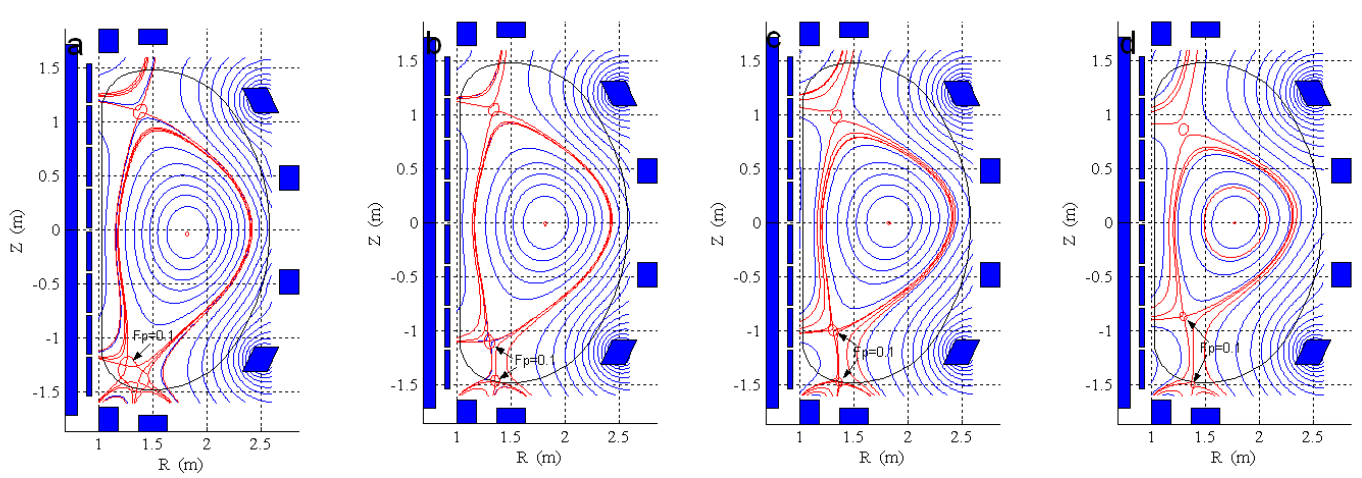

Figure 2. Configurations with different plasma currents; a) $I_{P}=2 \mathrm{MA}$, snowflake-minus divertor configuration, b) $I_{P}=1.5 \mathrm{MA}$, c) $I_{P}=1.2 \mathrm{MA}$ and d) $I_{P}=0.9 \mathrm{MA}$. Configurations b-d are the "tripod" configurations; they have some attributes of cusp and $\mathrm{X}$-divertor configurations.

To create these advanced divertor configurations for exploration in HL-2M, asymmetric divertor target geometries compatible with the standard and advanced divertor configurations are proposed. To predict the details of the scrape-off layer (SOL) and divertor plasma with different divertor configurations, two-dimensional numerical calculations with the SOLPS5. ${ }^{[12]}$ code have been performed taking into account the geometry of the HL-2M divertor. From the simulation model, it is found that the advanced divertors have longer connection lengths and larger plasma-wetted areas. When $P_{S O L}=10 \mathrm{MW}$ and $n_{\text {sep }}=2.5 \times 10^{19} / \mathrm{m}^{3}$, the peak heat load on the inner and outer target plates of standard divertor is $7.56 \mathrm{MW} / \mathrm{m}^{2}$ and $1.1 \mathrm{MW} / \mathrm{m}^{2}$ respectively. For snowflake-minus and tripod divertors, the heat load on the outer target plates will be substantially 
reduced with a broadened heat load profile, but more power will be transported to the inner divertor target plate and causes a higher peak heat load there. When the configuration is changed to a double-null tripod divertor with a different plasma current, then the peak heat load on the target plate is less than $4 \mathrm{MW} / \mathrm{m}^{2}$ with $P_{S O L}=20 \mathrm{MW}$, which is acceptable for the HL-2M divertor engineering design. The advanced divertor configurations of HL-2M will reduce the heat load on the target plates, but the open divertor structure will cause the divertor to operate under low recycling conditions, with high plasma temperature and low plasma density. Following heat load mitigation by optimizing the configuration and target geometry, the control and enhancement of particle recycling in the divertor region will also be an important issue in the divertor design of HL-2M.

\section{The analysis of pedestal instabilities in snowflake configurations}

In order to study the impact of the various divertor configurations on the power deposition during ELM bursts, the linear P-B mode (which is considered to be the dominant instability to trigger an ELM) is analyzed by the BOUT++ code. The four configurations shown in figure 1 have the same H-mode pressure and current profiles and the same flux surface at $\Psi<0.95$ (where $\Psi$ is the normalized poloidal flux, changing from 0 at the magnetic axis to 1 at the separatrix). The linear growth rate of the P-B mode is investigated and shown in figure 3. All four cases have the typical ballooning-dominant linear growth rate. The growth rate increases with the increase of toroidal mode number $\mathrm{n}$, although the high-n modes can be stabilized by ion diamagnetic drift. The P-B modes have similar growth rates for both the standard divertor configuration and the snowflake plus class. The exact snowflake configuration yields a growth rate around 5\% lower than that of the standard case. The snowflake-minus shows the lowest growth rate for P-B modes, around 30\% lower than that of the other configurations. Therefore we can conclude from Fig. 3 that the snowflake-minus configuration has the most stabilizing effect on the P-B modes, while the other configurations considered reveal almost no variation in the P-B modes.

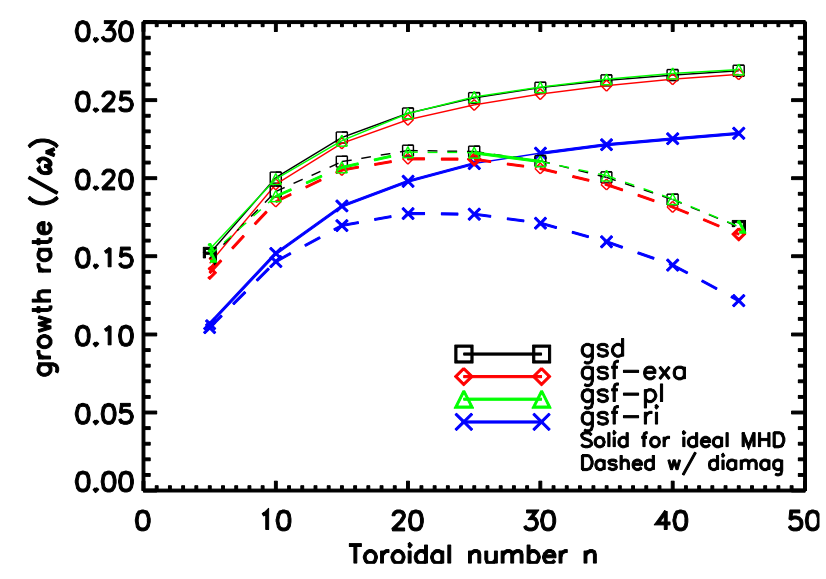

Figure 3. The linear growth rate of the Peeling-Ballooning mode for divertor geometries with the same equilibrium pressure. The black squares represent the standard divertor, red diamonds the exact snowflake, green triangles the snowflake plus, and blue crosses the snowflake-minus right. The solid curves are ideal MHD results neglecting diamagnetic effects and the dashed ones include the diamagnetic effects. The linear analysis shows that the snowflake-minus geometry has the lowest P-B instability. 
The reason for the different impact of the divertor configurations on the P-B modes is the finite magnetic shear, which has a stabilizing effect on ballooning modes. Figure 4 (a) shows the poloidal profile of the local magnetic shear at the peak pressure gradient position for the four configurations. The local magnetic shear is defined as

$$
\mathrm{S}=\frac{r}{v} \frac{\partial v}{\partial r}, \quad v=\frac{r B_{t}}{R B_{p}}
$$

Here $v(\Psi, \theta)$ is the local pitch and its flux surface average is the safety factor, $\mathrm{q}(\Psi)=\langle v\rangle_{\text {sur }}$. In the four types of equilibrium configurations, with the outer mid-plane located at $y$ index 43 (where the y-index is related to the distance along the last closed flux surface from the inboard target plate, see figure 4a), from figure 4 (a) we can find where the local shear of the standard divertor is smallest, and the exact snowflake has the largest local shear at the outer mid-plane where the ballooning modes develop. The differences between the four configurations are very small, so the linear growth rates for the standard, exact and snowflake plus classes are quite close. However, the local magnetic shear for both snowflake plus and minus is similar, which appears to be inconsistent with figure 4 (b) where it is shown that the global shear of snowflake-minus is much higher than the other three cases. The global shear is written as

$$
\mathrm{S}=\frac{r}{q} \frac{\partial q}{\partial r}
$$

Compared to the local shear s, the integrated magnetic shear $\mathrm{S}$ brings the null-point into consideration through the flux surface average operator. The snowflake-minus has the closest $\mathrm{X}$-point to the outer mid-plane and the large low field (meaning also the large magnetic shear) region is able to provide a stabilizing impact on ballooning modes. The second reason is that the snowflake-minus geometry tested here has the closest second X-point to the bad curvature region. In this case, the second X-point lessens the bad curvature on the low field side as shown in figure 5 and this is also contributes to stabilizing the P-B modes.
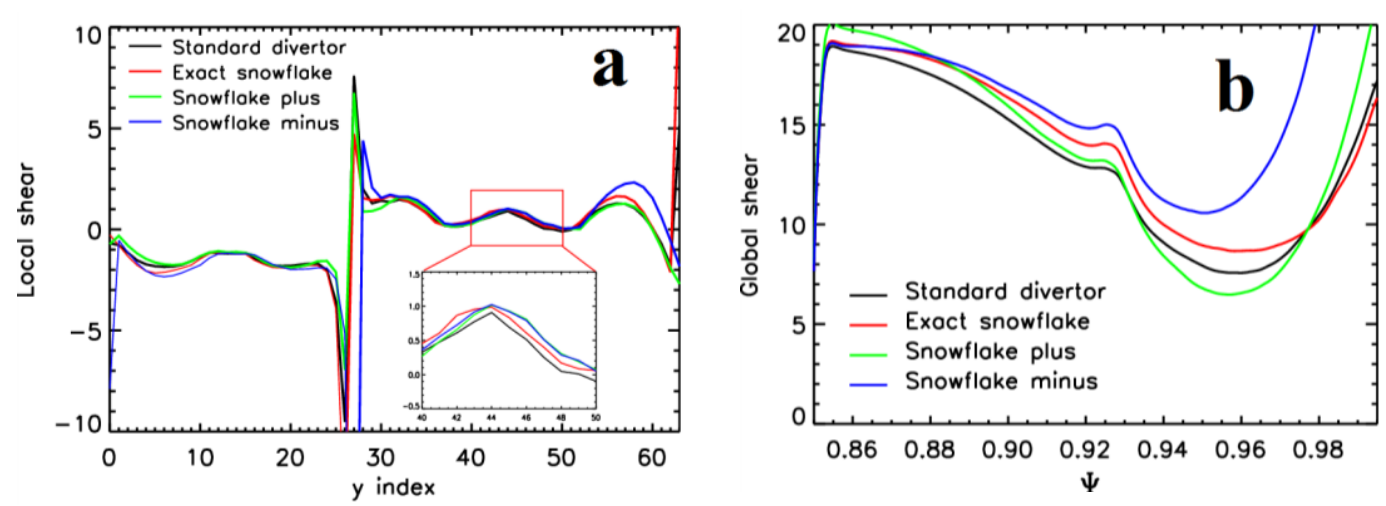

Figure 4. a) The local shear of four different divertor geometries. b) The integrated magnetic shear of these configurations. The instability of standard, exact SF and SF-plus geometries is correlated with the local shear at the outer mid-plane (where the y index is 43). The larger shear generally leads to smaller growth rate. However, the snowflake-minus behavior is related to the global shear which is changed because the second X-point affects the curvature at outer mid-plane. 


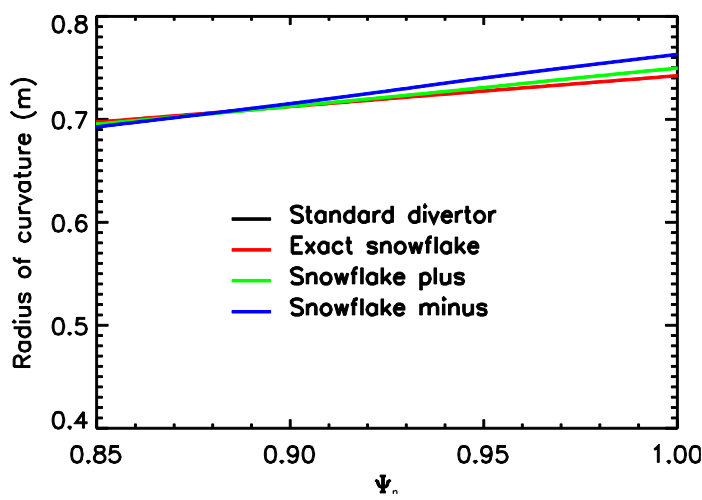

Figure 5. The comparisons of the radius of curvature on the mid-plane at low field side for the four different geometries. At the region where the ballooning modes develop, $\Psi \sim 0.96$, the snowflake-minus shows a larger radius of curvature than the other three cases. As a result, the bad curvature of the snowflake-minus is more improved by the presence of the second X-point than the other cases.

ELMs are here considered to be an ideal MHD P-B instability driven by edge gradients and stabilized by the magnetic shear. From the analysis produced by the BOUT++ code, it is found that the snowflake-minus can improve the stability of the P-B modes. The nonlinear process and the ELM sizes for these configurations will be studied considering the effect of the second X-point as well as the transient heat load to the target plate with a longer connection length, larger plasma wetted-area and increased cross-field plasma transport in the low $B_{p}$ zone around the X-points. The design of the divertor target geometry is being developed to ensure full compatibility with the intended range of configurations of HL-2M.

\section{Target design and simulation model}

Reducing the heat load on the divertor target and enhance the particle control ability are the main aims in the design of the HL-2M divertor. An asymmetric divertor target geometry is proposed which is compatible with the configurations shown above. In the standard divertor configuration shown in figure 6 (a), the lower divertor target geometry with inner and outer vertical target plates and a dome baffle in the private flux region has been designed with minimized conductance for neutral particle leakage from the divertor region into the main chamber ${ }^{[13]}$, and with a ' $\mathrm{V}$ ' shape similar to the ITER divertor, to further reduce the heat load ${ }^{[14,15]}$. The upper target plate is designed to suit the advanced divertor configurations shown in figure 1 (inverted) and figure 6 (b) and is therefore different from the lower target geometry. This is necessary to avoid adverse effects on the lower SN standard divertor configuration operation and to allow the second X-point in the advanced divertor to be close to the target plate to form a larger plasma-wetted area with $I_{P} \geq 2.0 \mathrm{MA}$. The upper target plate is designed to be close to the vacuum vessel (VV), conferring a large space for flexibility in the advanced divertor operation. When the second $\mathrm{X}$-point is close to the outer target plate in the snowflake-minus divertor configuration, the $F_{t}\left(=B_{p} / B_{t}\right)<0.02$ region will cover a small part of the target plate near the second X-point shown in figure 7 . Thus even with $\alpha$ close to $\pi / 2$ with an open divertor geometry of snowflake divertor, $\gamma_{\min }$ will still be less than 0.02 (i.e. roughly 1 degree) ${ }^{[7]}$ on the outer divertor region near the second X-point. It is required to avoid shadows and hot spots which may appear on the plate and this can be achieved by controlling the position of the second X-point above the target plate in HL-2M. Highly accurate 
configuration control technology for the second X-point of the advanced divertor operation is under development. The primary X-point and the inner strike point will move upward with the second X-point being located at the same place as for snowflake-minus, shown in figure 2. To ensure the inner strike point is on the inner target plate, a long inner target plate is designed for the tripod divertor configurations. In addition, HL-2M has a flexible PF coil control system to accommodate the DN standard, snowflake and tripod divertor configurations over a wide range of plasma currents. As shown in figure 6 (c), when the DN tripod divertor configuration is achieved in HL-2M, the lower divertor dome baffle will have a similar function to that of the lower-outer divertor target plate. In this way, the DN tripod divertor configurations will also be compatible with the divertor target geometry and can be tested in HL-2M.
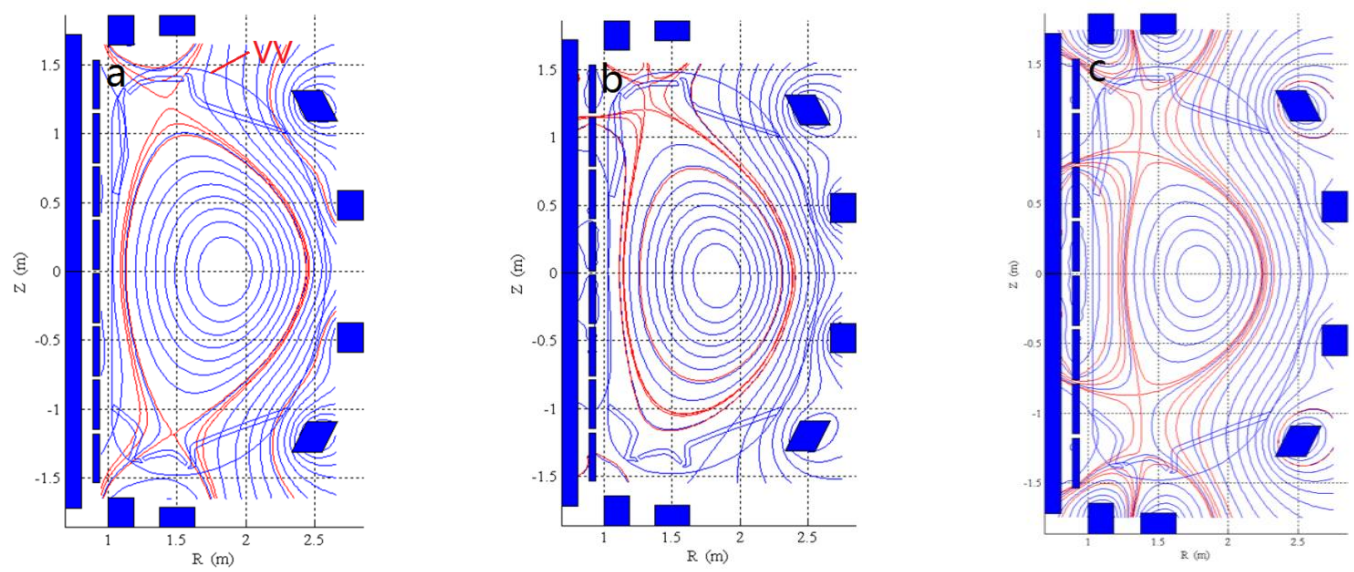

Figure 6. The asymmetric divertor target plate geometry; a) $I_{P}=2.0 \mathrm{MA}$, standard divertor configuration, b) $I_{P}=2.0 \mathrm{MA}$, snowflake-minus divertor configuration c) $I_{P}=0.9 \mathrm{MA}, \mathrm{DN}$ tripod divertor configuration. The first wall is not shown here and the distance from the primary X-point to the VV is about $30 \mathrm{~cm}$. The slot close to the VV for the neutralized particles is estimated to be about $3 \mathrm{~cm}$ wide and offers a pumping rate of $50 \mathrm{~m}^{3} / \mathrm{s}$ to the cryopump.

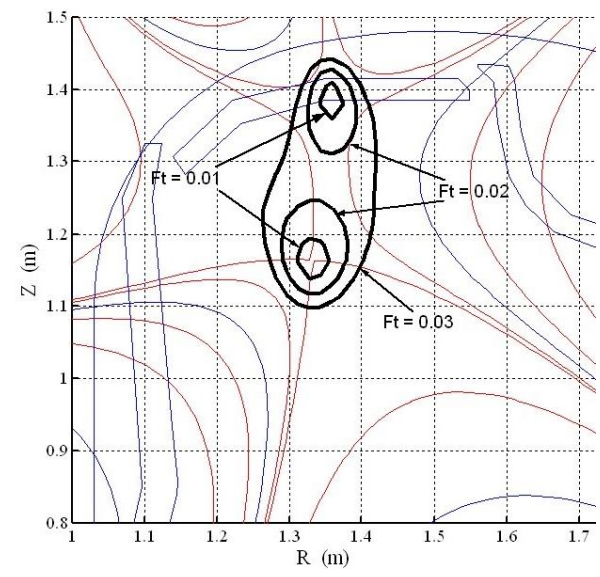

Figure 7 . The $F_{t}$ value of snowflake-minus around target plate.

Excessive heat load on the divertor plates would result in melting and evaporation, thus reducing discharge performance, and is sensitively dependent on the divertor/SOL plasma conditions. The heat load on the divertor target plates has been investigated for the HL-2M parameters by 
SOLPS5.0 (B2.5-Eirene). The B2.5-Eirene code package consists of a multi-fluid code B2.5 for electrons and ions at each ionization state, coupled to a Monte-Carlo code Eirene for neutrals. Mitigation of the heat loades on the target plates using only variations in the magnetic geometry is studied. $\mathrm{D}^{0}$ and $\mathrm{D}^{+}$plasma species are simulated in the divertor geometry to investigate the heat load on the target plate for different divertor configurations. With respect to cross-field transport, there still exist relatively large uncertainties, and here a constant cross-field diffusion coefficient $D$ $=0.15 \mathrm{~m}^{2} / \mathrm{s}$ and constant cross-field ion and electron heat diffusivities $\chi_{e}=\chi_{i}=0.5 \mathrm{~m}^{2} / \mathrm{s}$ are used $^{[2,16,17]}$ to predict the heat load on target plate by SOLPS5.0. At the Core-SOL interface (a few centimeters inside the separatrix), the density and the total power $P$ (equally split between the electron and ion heat channels in the computational region) across the Core-SOL boundary are described as common value in the input parameters. $n_{\text {sep }}$ is the separatrix density at outer mid-plane. In this model, the net neutral deuterium flux at the Core-SOL boundary interface is set to zero. The recycling coefficient for deuterium is set to $100 \%$ at the divertor target plates and the main chamber wall. The pumping port is taken to be in the private flux region.

\section{Simulation results}

When $n_{\text {sep }}=2.5 \times 10^{19} / \mathrm{m}^{3}$ which is about $30 \%$ of the line average density (the Greenwald density limit is $1.5 \times 10^{20} / \mathrm{m}^{3}$ when $I_{P}=2.0 \mathrm{MA}$ ) and $P_{S O L}=10 \mathrm{MW}$ with the configuration shown in Figure 6 (a), the heat load profiles on the inner and outer target plates of the standard divertor are shown in figure 8 . The peak heat load on the inner target plate is about $1.1 \mathrm{MW} / \mathrm{m}^{2}$ with low plasma temperature and high plasma density. More power is transported to the outer divertor region with the high parallel heat conduction, the electron temperature showing little drop along the field lines at the lower-outer target plate. The outer divertor operates in the low recycling and low plasma density regime, so that recycling losses can be neglected. Accordingly the peak heat load on the outer target plate is about $7.56 \mathrm{MW} / \mathrm{m}^{2}$, which is spread over a small area near the separatrix. The peak heat loades on the target plates with different powers flowing into the SOL zone are shown in figure 9. When $P_{S O L}=12 \mathrm{MW}$, the peak heat load on the outer target plate is about $10 \mathrm{MW} / \mathrm{m}^{2}$, and it is about $17.0 \mathrm{MW} / \mathrm{m}^{2}$ when $P_{S O L}=18 \mathrm{MW}$. HL-2M is designed with a total heating power of 25MW. From the engineering design point of view, it will be a challenge for HL-2M to operate with the peak heat load on the target plate beyond $10 \mathrm{MW} / \mathrm{m}^{2}$ under standard divertor operation. In the simulation carried out above, impurities were not included, such as carbon sputtering or impurity seeding. As the heat load is sensitive to plasma density, a larger $n_{\text {sep }}$ value is also required to reduce the heat load during high heating power operation.

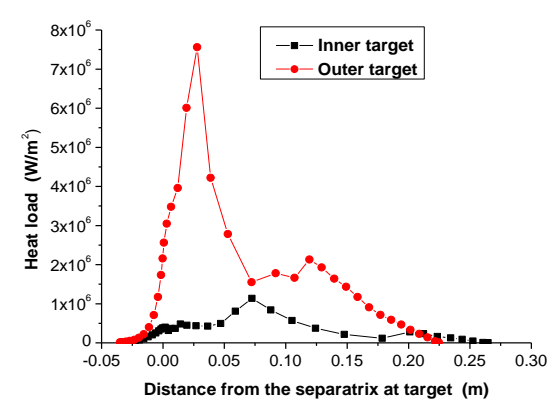




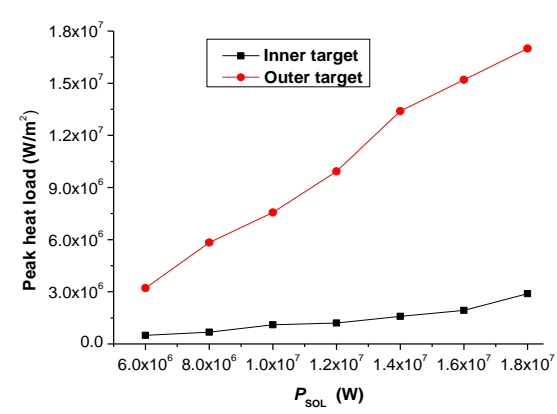

Figure 9. The peak heat load on target plates of standard divertor with varied $P_{S O L}$.

From figure 8, we find that the peak heat load is focused on a small area of the target plate near the separatrix in the standard divertor case. Compared to the standard divertor configuration, the snowflake-minus shown in figure 6 (b) has a second X-point close to the separatrix to form a larger low $B_{p}$ area and magnetic flux surface expansion, as well as a longer connection length. Connection length here means the length along the magnetic field line from the outer mid-plane to the outboard divertor target plate. The surface expansion and connection length are shown in figure 10 and figure 11, plotted against the distance of the respective flux surfaces from the separatrix at the outer mid-plane. In figure 11, the connection length is normalized to the connection length of the standard divertor configuration at a point which is $0.04 \mathrm{~mm}$ away from the separatrix at outer mid-plane. From tokamak experiments and theory ${ }^{[2,18]}$, it is found that most of $P_{S O L}$ is transported to the divertor target plates with a heat load width $\lambda_{q}$ which will be less than $5 \mathrm{~mm}$ at the outer mid-plane of HL-2M. The flux surface expansion and connection length of the snowflake divertor are much larger than that of the standard divertor, especially at the point with $\lambda_{q}$ less than $2 \mathrm{~mm}$, and both of these increases are expected to reduce the heat load on the divertor target by broadening the heat load profile ${ }^{[19]}$. With the same computational boundary plasma conditions as in the standard divertor simulated above, the snowflake-minus divertor shown in figure 6 (b) is simulated by SOLPS5.0. The heat load profiles are shown in figure 12. The peak heat load on the outer target plate decreases to about $3.5 \mathrm{MW} / \mathrm{m}^{2}$, and is located at the place which is $15 \mathrm{~cm}$ away from the separatrix. Due to the open divertor target plates adopted for the snowflake-minus configuration, intended to generate a larger plasma-wetted area, the neutrals produced on the target plates are reflected towards the core region and ionization is not enhanced near the vicinity of the separatrix, which results in the outer divertor working under a low recycling regime with high electron temperature. For the inner divertor, the distance between the $\mathrm{X}$-point and the inner target plate is about $20 \mathrm{~cm}$, and more power will be transported to the inner target with high parallel heat conduction. The peak heat load is shown to be $8.3 \mathrm{MW} / \mathrm{m}^{2}$ over a small area near the separatrix with the electron temperature close to that at the outer mid-plane. Figure 13 shows that the peak heat load on the target plates of snowflake-minus divertor. $P_{\text {inner.target }}$ is the power fraction transported to the inner target plate. $P_{\text {inner.target }} / P_{S O L}$ is about $10 \%$ of that of the standard divertor from the simulation results, which leads to the peak heat load on the inner divertor target plate being much lower than that on the outer divertor target plate. But for the snowflake-minus divertor, $P_{\text {inner.target }} / P_{S O L}$ is more than $40 \%$ with $P_{S O L}$ from 
$6 \mathrm{MW}$ to18MW, which results in a higher peak heat load on the inner target plate. When $P_{S O L}=$ $18 \mathrm{MW}$, the peak heat load on the inner target is about $20.0 \mathrm{MW} / \mathrm{m}^{2}$. For the outer divertor target, owing to the larger plasma-wetted area and longer connection length, the profiles will become flat and the peak heat load on the target plate is about $6.5 \mathrm{MW} / \mathrm{m}^{2}$ with $P_{S O L}=18 \mathrm{MW}$. Hence for the snowflake-minus divertor of HL-2M, the peak heat load may be reduced on the outer divertor target plate, but the open target geometry cannot keep the neutrals produced at the target plates near the vicinity of the separatrix, which results in operating in the low recycling regime with high particle temperature and low particle density. The peak heat load on the outer target plate of the standard and snowflake-minus divertors with different $n_{\text {sep }}$ are shown in figure 14. When $P_{S O L}=$ $10 \mathrm{MW}$, the peak heat load on the outer target plate of the standard divertor increase strongly with $n_{\text {sep }}$ decreasing, reaching $10.0 \mathrm{MW} / \mathrm{m}^{2}$ with $n_{\text {sep }}=1.8 \times 10^{19} / \mathrm{m}^{3}$. For the snowflake-minus divertor, the peak heat load on the outer target plate is not sensitive to $n_{\text {sep }}$ with the heat load profile becoming more flatten when $n_{\text {sep }}$ decreases, but the peak heat load on the outer target plate increases obviously, it is about $11.2 \mathrm{MW} / \mathrm{m}^{2}$.

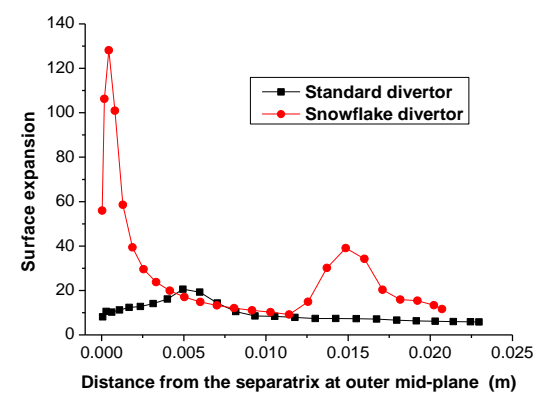

Figure 10. Surface expansion on the target plates of the standard and snowflake-minus divertors.

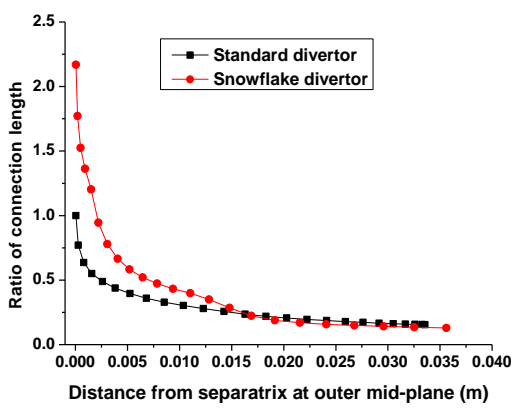

Figure 11. Ratio of connection lengths of the standard and snowflake-minus divertor.

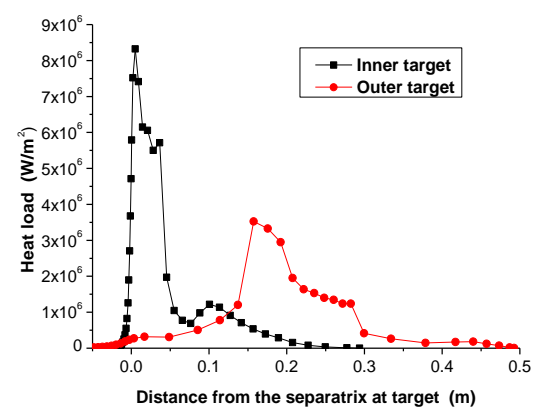


Figure12. Heat load profiles on the target plates of the snowflake-minus divertor.

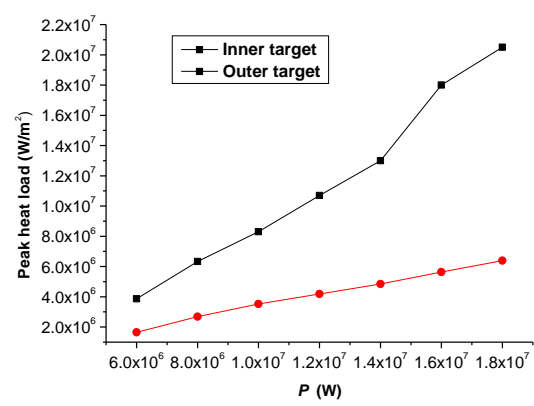

Figure 13. The peak heat loads on the target plates of the snowflake-minus divertor.

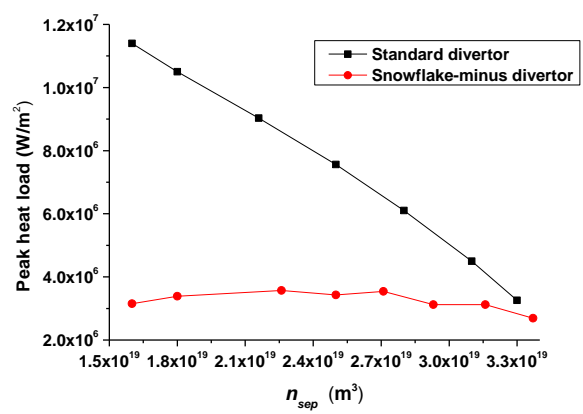

Figure 14. The peak heat loads on the outer target plates with different $n_{\text {sep }}$ of the standard and snowflake-minus divertors.

The tripod divertor configurations and associated upper target plates are shown in figure 15 . The tripod divertor configurations have the second X-point close to the outer target plate to form a larger plasma-wetted area and longer connection length than in the standard divertor, similar to the snowflake-minus divertor case, but the outer divertor leg is longer than that of the snowflake-minus. If the $\lambda_{q}=1.5 \mathrm{~mm}$ for $I_{P}=2.0 \mathrm{MA}$ is considered in this paper, then values of $\lambda_{q}$ of $I_{P}=1.5 \mathrm{MA}, I_{P}=1.2 \mathrm{MA}$ and $I_{P}=0.9 \mathrm{MA}$ correspond to about $2.1 \mathrm{~mm}, 3 \mathrm{~mm}$ and $4.5 \mathrm{~mm}$ respectively. The transport factor constants are adjusted in the modeling to achieve this variation. When $n_{\text {sep }}=2.0 \times 10^{19} / \mathrm{m}^{3}$ and $P_{S O L}=10 \mathrm{MW}$, the simulation results from SOLPS5.0 shows that the tripod divertors behave like the snowflake, and more power will be transported to the inner target with high parallel heat conduction. This results in a high heat load with low particle recycling and high electron temperature, close to that of the outer mid-plane. The peak heat load on the inner target plates is about $10.0 \mathrm{MW} / \mathrm{m}^{2}$ when $I_{P}=1.5 \mathrm{MA}$ and $I_{P}=1.2 \mathrm{MA}$, and it is $7.56 \mathrm{MW} / \mathrm{m}^{2}$ when $I_{P}=0.9 \mathrm{MA}$. The heat load profiles on the outer divertor target plate becomes flat, then the peak heat load is less than $2.2 \mathrm{MW} / \mathrm{m}^{2}$. 

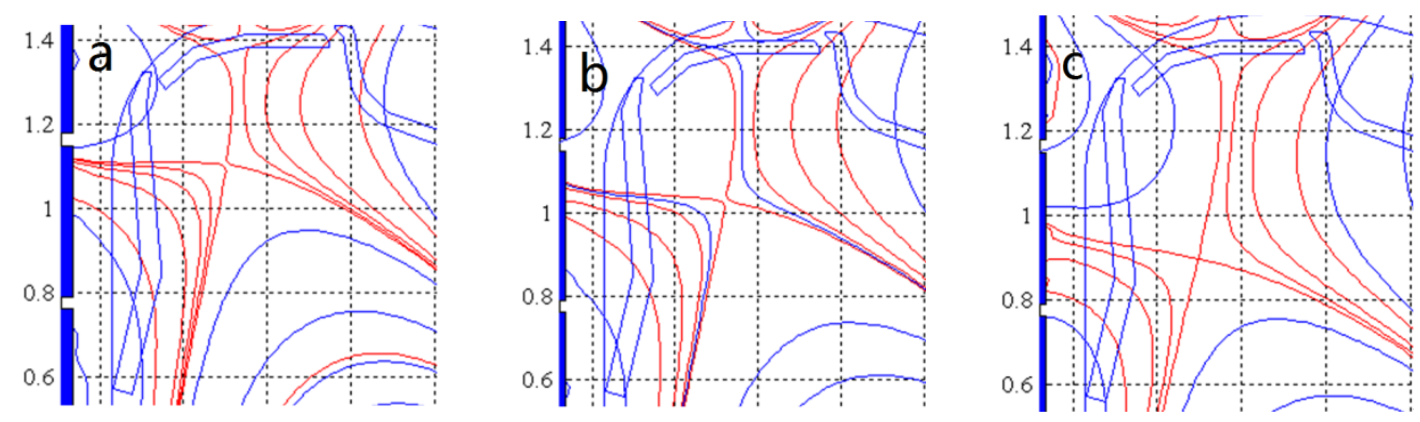

Figure 15. Tripod divertor configurations, a) $\left.\left.\mathrm{I}_{\mathrm{P}}=1.5 \mathrm{MA}, \mathrm{b}\right) I_{P}=1.2 \mathrm{MA}, \mathrm{c}\right) I_{P}=0.9 \mathrm{MA}$. The second X-point and the outer target strike point is almost at the same place, but the primary X-point and inner strike point will move down when the plasma current reduces, so the distance between the two X-points along the separatrix, $d_{X}$, will increase. When $I_{P}=0.9 \mathrm{MA}, d_{X}$ is more than $50 \mathrm{~cm}$.

From the heating deposition point of view, high auxiliary heating power operation will cause a high heat load on the outer target plate with the standard divertor and a high heat load on the inner target plate with the snowflake and tripod divertors of the SN divertor configurations. In order to handle $P_{S O L}=20 \mathrm{MW}$ without impurity seeding, the DN tripod divertor configurations are considered as an effective means to reduce the peak heat load on the target plate to an acceptable level in HL-2M configurations and target geometry design. As the outer SOL zone has a larger surface area, and the magnetic flux surfaces are compressed with respect to the inboard side due to the Shafranov shift, there will be a stronger radial gradient. This would result in an enhanced cross-field heat load into the outer SOL zone if the cross field transport is dependent on spatial gradients. So the power crossing the Core-SOL boundary interface is assumed to be distributed between the outer SOL zone and the inner SOL zone of the DN tripod configuration with a ratio of 3 to investigate the heat load on the target. As result, $25 \%$ of the power flows into the inner SOL zone and is divided between the two inner target plates to reduce $P_{\text {inner.target }} / P_{S O L}$ and the peak heat load on the inner target plates. $75 \%$ of $P_{S O L}$ will be handled by the outer divertor target plates with a longer connection length and a larger plasma-wetted area. The DN tripod configurations with different plasma currents are shown in figure 16.
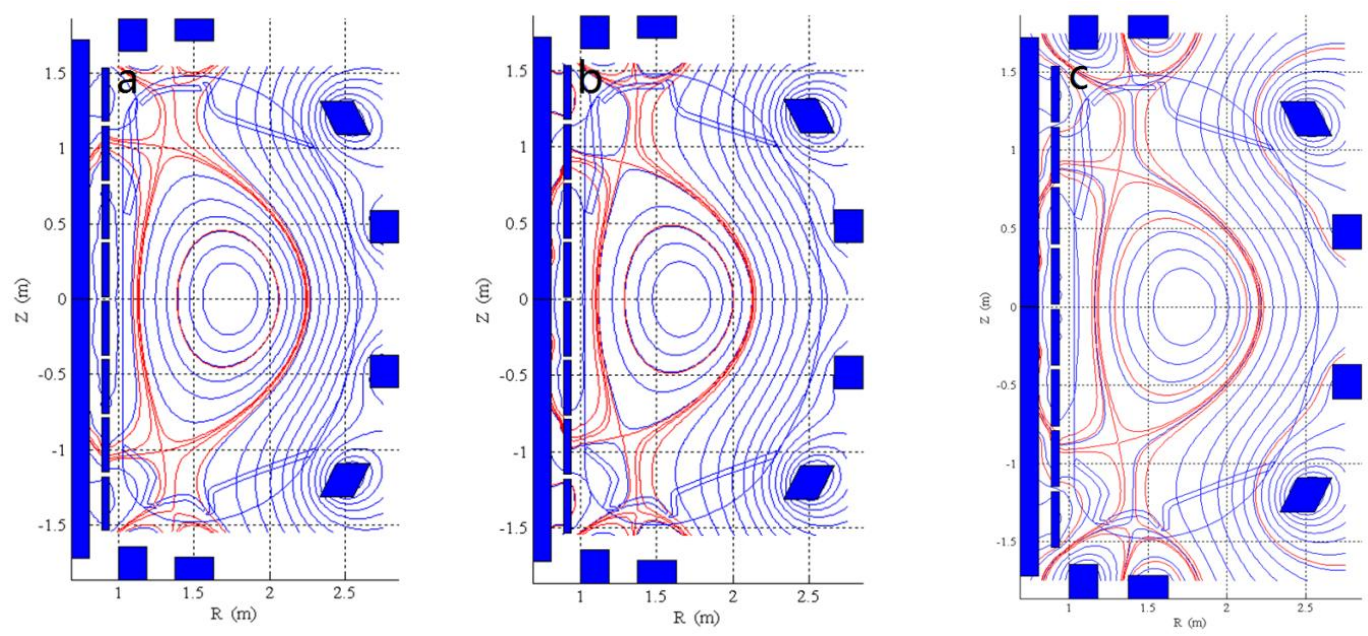
Figure 16. DN tripod divertor configurations. a) $I_{P}=1.2 \mathrm{MA}$, b) $I_{P}=0.9 \mathrm{MA}$, c) $I_{P}=0.7 \mathrm{MA}$. The upper second $\mathrm{X}$-point is designed to be close to the upper-outer divertor target plate with a large surface expansion, but the lower second X-point is about $6 \mathrm{~cm}$ far away from the target plate, causing the magnetic flux expansion at the target plate and the connection length from the outer mid-plane to the lower-outer target to reduce.

For DN tripod divertor configurations, constant cross-field transport factors $D=0.3 \mathrm{~m}^{2} / \mathrm{s}$ and $\chi_{e}=$ $\chi_{i}=1.0 \mathrm{~m}^{2} / \mathrm{s}$ are used. When $n_{\text {sep }}=1.4 \times 10^{19} / \mathrm{m}^{3}$ and $P_{S O L}=10 \mathrm{MW}$, the heat load profiles on the target plates of the three divertor configurations are shown in figure 17. As the heat load profiles on the upper-inner and lower-inner targets depicted in figure 17 (a) and figure 17 (c) show, the peak heat loades are located at the point near the separatrix, but their values are less than $2.0 \mathrm{MW} / \mathrm{m}^{2}$. For the upper-outer divertor, the second X-point is designed to be near the upper-outer divertor target plate with a larger plasma-wetted area and longer connection length, so that the heat load profiles are flat, as shown in figure 17 (b) with the peak heat load on target plate less than $1.0 \mathrm{MW} / \mathrm{m}^{2}$. The heat load profiles at the lower-outer target plates are shown in figure $17(\mathrm{~d})$. The peak heat load on the upper-outer target plate is less than $1.8 \mathrm{MW} / \mathrm{m}^{2}$, but the heat load profiles on the targets are little peaked compared to those of the upper-outer divertor due to the different distance between the second X-point and the target plate. Thus DN tripod divertor configurations will enable HL-2M to handle greater power exhaust flows into the SOL zone with the peak heat load on the target plate within the engineering design limits. Taking the configurations shown in figure 17 (c) for example, when $P_{S O L}=20 \mathrm{MW}$, the peak heat loads on the upper-inner and lower-inner target plates are $4.0 \mathrm{MW} / \mathrm{m}^{2}$ and $3.2 \mathrm{MW} / \mathrm{m}^{2}$ and on the upper-outer and lower-outer target plates are $1.7 \mathrm{MW} / \mathrm{m}^{2}$ and $4.1 \mathrm{MW} / \mathrm{m}^{2}$.
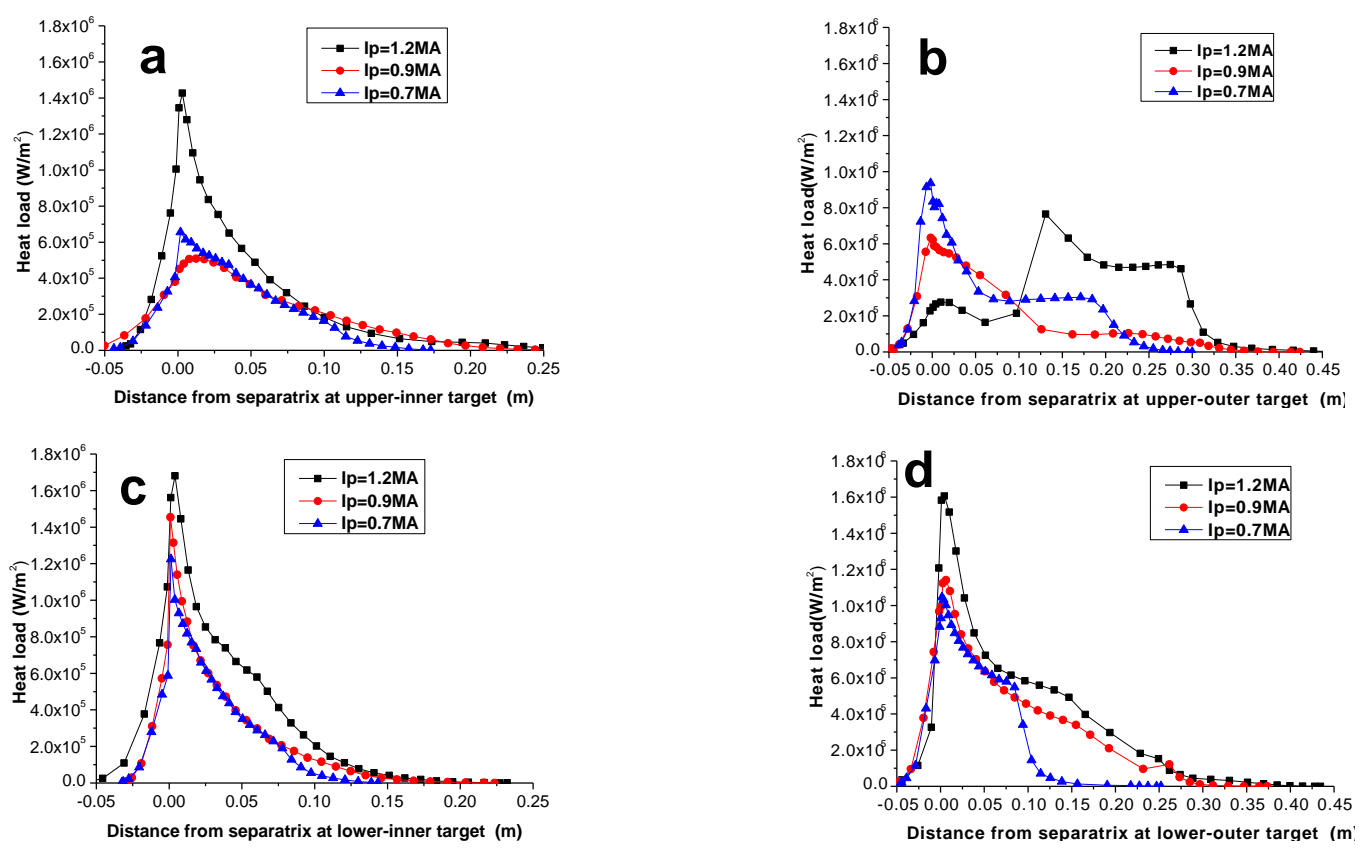

Figure 17. The heat load profiles on the target plates of the DN tripod divertor configurations, a) Heat load profiles at the upper-inner divertor target plate, b) Heat load profiles at the upper-outer divertor target plate, c) Heat load profiles at the lower-inner divertor target plate, d) Heat load profiles at the lower-outer divertor target plate. 
The HL-2M PF coil system is designed to be very flexible in order to control the plasma configuration, including the positions of primary and second X-points to suit the experimental requirements. The target plates are designed to let the second X-point appear above the target plate for the snowflake and tripod divertor configurations. From the simulation above, the second $\mathrm{X}$-point of the HL-2M advanced divertor is not included, and the influence of the second X-point is not well estimated by SOLPS5.0, especially the particle density, particle temperature and the share of the power exhaust through the second X-point near the divertor target. When the two $\mathrm{X}$-points get close enough to form a larger low $B_{p}$ area as in the exact snowflake divertor, fast convective heat transport around the two X-points is expected and should represent another important way to mitigate the heat load on the target plates by increasing the power sharing among the four divertor legs and by broadening the heat load profile on the divertor target plates to reduce the peak heat load during ELM bursts.

\section{Summary}

Based on the CS and PF coil system design of HL-2M, the standard, snowflake and tripod divertor configurations have been designed. The stability of the P-B mode is analyzed by the BOUT++ code for snowflake divertors in HL-2M, the results showing that snowflake divertors will reduce the linear growth rate of the P-B modes by increasing the magnetic shear and lessening the bad curvature at low field side with the second X-point, especially in the snowflake-minus divertor configuration. The ELM size and transient heat load on target plate due to ELM bursts will be further studied, coupled with various divertor configurations and target geometries in HL-2M. According to the properties of these divertor configurations ${ }^{[3]}$, asymmetric divertor target plates arranged to be compatible with these divertor configurations are proposed. The divertor plasma in these divertor configurations has been simulated by SOLPS5.0 for a pure deuterium plasma. For the standard divertor when $n_{\text {sep }}=2.5 \times 10^{19} / \mathrm{m}^{3}$, the peak heat load on the outer target plate about $7.56 \mathrm{MW} / \mathrm{m}^{2}$ with $P_{S O L}=10 \mathrm{MW}$, and increases to about $10 \mathrm{MW} / \mathrm{m}^{2}$ and $17 \mathrm{MW} / \mathrm{m}^{2}$ with $P_{S O L}=$ $12 \mathrm{MW}$ and $P_{S O L}=18 \mathrm{MW}$. As the heat load on the target is sensitive to $n_{s e p}$, increasing the plasma density will be an important way to mitigate the heat load in high power operation. For the snowflake-minus and tripod divertors, the heat load on the outer target plates will be substantially reduced by the larger plasma-wetted area and longer connection length which will broaden the heat load profile, but more power will be transported to the inner divertor target plate and there will be a higher peak heat load on the inner target plate. When $P_{S O L}=10 \mathrm{MW}$ and $P_{S O L}=18 \mathrm{MW}$ in the snowflake-minus $\left(I_{P}=2.0 \mathrm{MA}\right)$, the peak heat load on the inner targets is about $10.7 \mathrm{MW} / \mathrm{m}^{2}$ and $20 \mathrm{MW} / \mathrm{m}^{2}$ respectively, but the peak heat load on the outer targets will be $3.5 \mathrm{MW} / \mathrm{m}^{2}$ and $6.5 \mathrm{MW} / \mathrm{m}^{2}$. Thus for the $\mathrm{SN}$ advanced divertor configurations, it may be possible to reduce the peak heat load on the outer divertor target plate, but there is likely to be a higher peak heat load on the inner target plate. Even if the peak heat load on the outer target plate is substantially reduced, but the outer divertor works under low plasma recycling condition with high particle temperature and low density as in the inner divertor situation, then improving the particle control will be a challenging issue for HL-2M advanced divertor operation. From the engineering cooling design point of view, it will be a challenge for the high power exhaust operation of the HL-2M SN divertor (neglecting possible favourable impurity effects). When HL-2M is changed to the DN tripod divertor configuration, considering the outer SOL zone has a larger surface area and higher 
pressure gradient, assuming that about $25 \%$ of the power exhaust flows into the inner SOL zone, then most of the power exhaust is handled by the outer target taking advantage of the larger plasma-wetted area and longer connection length. The results show that when $P_{S O L}=10 \mathrm{MW}$ and $P_{S O L}=20 \mathrm{MW}$, the peak heat load on the target plate is about $1.8 \mathrm{MW} / \mathrm{m}^{2}$ and $4.1 \mathrm{MW} / \mathrm{m}^{2}$, which is acceptable for the HL-2M divertor engineering design.

In the simulations above, the second X-point of the advanced divertor is not included in the model, and the influence of the second X-point is not well estimated by SOLPS5.0. The simulation result shows that the advanced divertor will reduce the heat load as a result of the larger plasma-wetted area and longer connection length, but it is expected to operate under a low recycling regime with high particle temperature and low particle density. If the carbon sources from the first wall and target plate (physical sputtering and chemical sputtering) or impurity seeding is included in the divertor simulation, the power balance in the divertor plasma should become dominated by the impurity distribution, especially in the advanced divertor with an open divertor geometry. Therefore, the appearance of the second X-point above the target for modeling with carbon sources or impurity seeding is expected to improve the particle recycling condition, reduce peak heat load and enhance the particle control ability of the HL-2M advanced divertor operation. As well as the larger plasma-wetted area and longer connection length of the snowflake-minus and tripod divertor configurations, the snowflake divertor configurations with a larger low $B_{p}$ area around the two X-points should create fast cross-field convective heat transport which will increase power sharing among the divertor legs and broaden the heat load profiles on the target plates and these effects are also expected in HL-2M experiments to mitigate the heat load.

\section{Acknowledgements}

The authors wish to acknowledge Drs. T.N. Todd and L.J. Cai for their valuable suggestions, advice and timely help for HL-2M divertor engineering design. Many thanks in particular to Drs. Y. Liu for his support and encouragement for HL-2M divertor design in SWIP. This work was supported by Chinese ITER Plan Project Foundation (Grant Nos. 2013GB113001, 2015GB105001 and 2013GB112009), National Science Foundation of China (grant Nos. 11575056 and 11275061), and by LLNL for USDOE under DE-AC52-07NA27344. LLNL-JRNL-651522.

\section{References}

[1] Q. Li, Proc. 25th Symposium on Fusion Engineering, TPO-113, 2013.

[2] T. Eich, A.W. Leonard, R.A. Pitts, W. Fundamenski, R.J. Goldston, T.K. Gray, et al., Nucl. Fusion 53 (2013) 093031.

[3] D.D. Ryutov, Phys. Plasmas 14 (2007) 064502.

[4] G.Y. Zheng, X.Q. Xu, D.D. Ryutov, Y.D. Pan, T.Y. Xia, Fusion Eng. Design 89 (2014) 2621-2627.

[5] L.D. Pearlstein, R.H. Bulmer, T.A. Casper, E.B. Hooper, R.A. Jong, T.B. Kaiser, et al., Proceedings 28th EPS Conference, P5.034, 2001.

[6] D.D. Ryutov, R.H. Cohen, T.D. Rognlien, M.V. Umansky, Contrib. Plasma Phys. 52 (5-6) (2012) 539543.

[7] A.S. Kukushkin, private communication to D. D. Ryutov (2007).

[8] J.F. Ma, X.Q. Xu, B.D. Dudson, Nucl. Fusion 54 (2014) 033011.

[9] M.V. Umansky, T.D. Rognlien, D.D. Ryutov, P.B. Snyder, Contrib. Plasma Phys. 50 (3-5) 
(2010) 350-355.

[10] M. Kotschenreuther, P.M. Valanju, S.M. Mahajan, J.C. Wiley, Phys. Plasmas 14 (2007) 072502 .

[11] H. Takase, J. Phys. Soc. Jpn. 70 (2001) 609.

[12] D.P. Coster, X. Bonnin, B. Braams, H. Buerbaumer, E. Kaveeva, J.W. Kim, et al., Proc. 19th IAEA Fusion Energy Conf., TH/P2-13, 2002.

[13] A.S. Kukushkin, H.D. Pacher,V.Kotov, D. Reiter, D. Coster and G.W. Pacher, Nucl. Fusion 47 (2007) 698-705.

[14] G. Janeschitz, A. Antipenkov, G. Federici, C. Ibbott, A. Kukushkin, P. Ladd, et al., Nucl. Fusion 42 (2002) 14-20.

[15] A.S. Kukushkin, H.D. Pacher, A. Loarte, V. Komarov, V. Kotov, M. Merola, et al., Nucl. Fusion 49 (2009) 075008.

[16] A.S. Kukushkin, H.D. Pacher, G.W. Pacher, V. Kotov, R.A. Pitts and D. Reiter, et al., Nucl. Fusion 53 (2013) 123024.

[17] A.S. Kukushkin, H.D. Pacher, G.W. Pacher, V. Kotov, R.A. Pitts and D. Reiter, J. Nucl. Mater. 438 (2013) S203-S207.

[18] T. Eich, B. Sieglin, A. Scarabosio, W. Fundamenski, R. J. Goldston, A. Herrmann and and ASDEX Upgrade Team, Phys. Rev. Lett. 107 (2011) 215001.

[19] V.A. Soukhanovskii, R.E. Bell, A. Diallo, S. Gerhardt, S. Kaye, E. Kolemen, et al., J. Nucl. Mater. 438 (2013) S96-S101. 